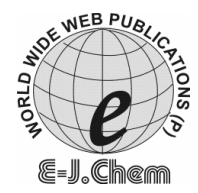

http://www.e-journals.net

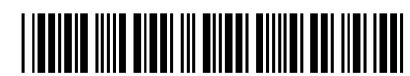

ISSN: 0973-4945; CODEN ECJHAO

E-Journal of Chemistry 2009, 6(3), 844-848

\title{
Synthesis and Antimicrobial Activity of Some New Formazan Derivatives
}

\author{
S. I. MARJADI, J. H. SOLANKI ${ }^{\S}$ and A. L. PATEL ${ }^{*}$ \\ ${ }^{\S}$ Department of Chemistry, Government College, Daman. \\ *Department of Chemistry, Faculty of Science, \\ The Maharaja Sayajirao University of Baroda, \\ Vadodara-390 002, India. \\ arunpatel_5376@yahoo.co.in
}

Received 1 October 2008; Accepted 11 December 2008

\begin{abstract}
A series of new substituted formazan derivatives has been synthesized from corresponding aryl diazonium chloride and Schiff base in pyridine. The synthesized compounds were identified by spectral studies and screened for their antimicrobial activities.
\end{abstract}

Keywords: Schiff base, Formazan, Antimicrobial activity.

\section{Introduction}

Formazans have been found to possess important medical applications; the tetrazolium salts are classified as promoter of vitality formazans and heterocyclic hydrazones are known for their spectrum of biological activities such as antiviral ${ }^{1,2}$, antimicrobial ${ }^{3}$, anti-inflammatory ${ }^{4}$, antifungal $^{5}$, anticancer ${ }^{6}$, anti-HIV ${ }^{7-8}$, etc. Several formazans show promising anti-fertility ${ }^{9}$ and anti-parkinsonian activity ${ }^{10-13}$.

Piperazine derivatives have also shown to possess diverse biological properties such as anti-histamine, antikatonic, anticonvulsant, antibacterial ${ }^{14}$ and as potential cocaineabuse therapeutic agents ${ }^{15}$. Piperazine possesses dopamine receptor activating property. The two nitrogen of the piperazine molecule are present in a rigid conformational structure that probably favours their involvement to the dopamine receptors ${ }^{16}$. In the present study we have synthesized ten substituted formazan derivatives (3a-j) by coupling Schiff base with appropriate aryl diazonium chlorides in pyridine. (Scheme 1) The structures of these derivatives were assigned on the basis of elemental analysis, IR and ${ }^{1} \mathrm{H}$ NMR spectral data. The synthesized compounds were screened for their antimicrobial activities. 


\section{Experimental}

Melting points were determined in open capillary tubes and are uncorrected. IR spectra were recorded in $\mathrm{KBr}$ on a Shimadzu $8400 \mathrm{~S}$ FTIR spectrophotometer. ${ }^{1} \mathrm{H}$ NMR spectra were recorded on a Hitachi $300 \mathrm{MHz}$ using TMS as an internal standard and elemental analysis had been carried out on Perkin-Elmer CHNS-2400.

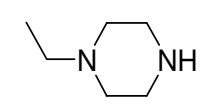

N-ethylpiperazine<smiles>Nc1ccc(Cl)cc1</smiles>

p-chloroaniline
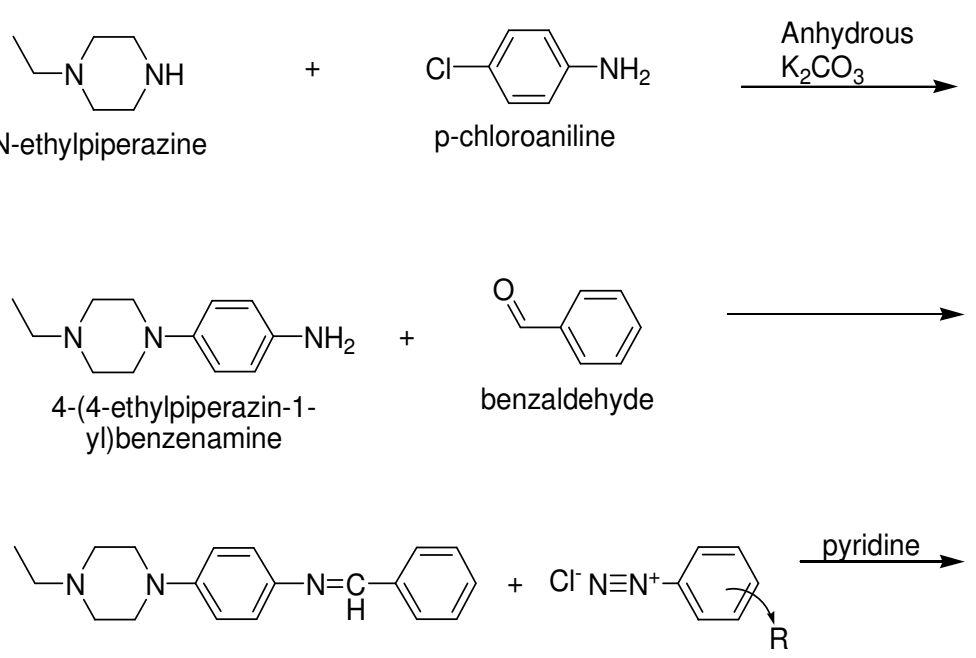

$N$-benzylidene-4-(4-ethylpiperazin-1- aryl diazonium chloride yl)benzenamine<smiles>CCN1CCN(c2ccc(N=C(N=Nc3cccc(P)c3)c3ccccc3)cc2)CC1</smiles>

Where $\mathrm{R}=-\mathrm{H}, 2-\mathrm{NO}_{2}, 3-\mathrm{NO}_{2}, 4-\mathrm{NO}_{2}, 4-\mathrm{Cl}$, 2- $\mathrm{Cl}, 4-\mathrm{OCH}_{3}, 2-\mathrm{OCH}_{3}, 4-\mathrm{Br}, 4-\mathrm{CH}_{3}$

\section{Scheme 1.}

\section{General procedure for the preparation of formazans}

Preparation of 4-(4-ethylpiperazin-1-yl) benzenamine (1)

A mixture of $N$-ethylpiperazine $(0.1 \mathrm{~mol})$ and $p$-chloroaniline $(0.1 \mathrm{~mol})$ and anhydrous $\mathrm{K}_{2} \mathrm{CO}_{3}$ in absolute alcohol $(20 \mathrm{~mL})$ was refluxed for $4 \mathrm{~h}$. The resultant mixture was cooled to room temperature and poured into ice-cold water with stirring. The separated solid was filtered, washed with water and recrystallized from ethanol.

\section{Preparation of N-benzylidene-4-(4-ethylpiperazin-1-yl) benzenamine (2)}

A mixture of 4-(4-ethylpiperazin-1-yl) benzenamine $(0.1 \mathrm{~mol})$ and benzaldehyde $(0.1 \mathrm{~mol})$ in absolute alcohol $(20 \mathrm{~mL})$ was refluxed for $2 \mathrm{~h}$. After the completion of reaction it was poured into ice-cold water with stirring. The solid product obtained was filtered, washed with water and recrystallized from ethanol. 
Preparation of $\mathrm{N}$-(Substituted phenylimino)-N'-(4-(4-ethylpiperazin-1-yl) phenyl) benzamidine (Formazans) (3a-j)

A cold stirred solution of aniline $(0.01 \mathrm{~mol})$ previously dissolved in aqueous $\mathrm{HCl}(10 \mathrm{~mL})$ was diazotized over crushed ice by dropwise addition of cold aqueous solution of $\mathrm{NaNO}_{2}$ $(2.5 \mathrm{~g})$ with stirring till a clear solution of diazonium salt of respective amine was obtained. The temperature ${ }^{17}$ was at $0-5{ }^{\circ} \mathrm{C}$. It was further stirred until the positive test of nitrous acid on starch-iodide paper was obtained. This mixture was then poured into a cold solution of $\mathrm{N}$ Benzylidene-4-(4-ethylpiperazin-1-yl) benzenamine $(0.01 \mathrm{~mol})$ dissolved in dry pyridine $(5.0 \mathrm{~mL})$. The reaction mixture was further stirred for $2 \mathrm{~h}$ maintaining temperature $0-5{ }^{\circ} \mathrm{C}$. The mixture was then poured into water with continuous stirring. The resultant dark coloured mass was filtered and washed with water till free from pyridine, dried and recrystallized from ethanol.

The spectral data of the synthesized compounds $\mathbf{3 a - j}$ are given below.

$N^{\prime}$-(4-(4-Ethylpiperazin-1-yl)phenyl)- $N$-(phenylimino)benzamidine (3a)

Yield 70\%, m.p. $47^{\circ} \mathrm{C}$. IR $\left(v, \mathrm{~cm}^{-1}\right)(\mathrm{KBr}): 1610,1520,1450,1390,1250 ;{ }^{1} \mathrm{H}$ NMR (DMSO): $\delta$ $1.13\left(\mathrm{t}, 3 \mathrm{H},-\mathrm{CH}_{3}\right), 2.4\left(\mathrm{q}, 2 \mathrm{H},-\mathrm{CH}_{2}\right), 2.56-3.52(\mathrm{t}, 8 \mathrm{H}$, piperazine), 6.6-7.3 (m, 14H, Ar-H); Anal. Calcd for $\mathrm{C}_{25} \mathrm{H}_{27} \mathrm{~N}_{5}$ : C, 75.57, H, 6.80, N, 17.62\%. Found C, 75.60, H, 6.83, N, 17.61\%.

$N$-(2-Nitrophenylimino)-N'-(4-(4-ethylpiperazin-1-yl)phenyl)benzamidine (3b)

Yield $65 \%$, m.p. $130{ }^{\circ} \mathrm{C}$. IR $\left(v, \mathrm{~cm}^{-1}\right)(\mathrm{KBr}): 1610,1580,1500,1460,1390,1320,1280,850$; ${ }^{1} \mathrm{H}$ NMR (DMSO): $\delta 1.1$ (t, 3H, - $\mathrm{CH}_{3}$ ), $2.4\left(\mathrm{q}, 2 \mathrm{H},-\mathrm{CH}_{2}\right), 2.59-3.45$ (t, 8H, piperazine), 6.6$8.2(\mathrm{~m}, 13 \mathrm{H}$, Ar- $\mathrm{H})$; Anal. Calcd for $\mathrm{C}_{25} \mathrm{H}_{26} \mathrm{~N}_{6} \mathrm{O}_{2}: \mathrm{C}, 67.89, \mathrm{H}, 5.88, \mathrm{~N}, 19.0 \%$. Found $\mathrm{C}$, $67.85, \mathrm{H}, 5.87, \mathrm{~N}, 19.02 \%$

$N$-(3-Nitrophenylimino)- $N^{\prime}-(4-(4-e t h y l p i p e r a z i n-1-y l)$ phenyl)benzamidine (3c)

Yield 65\%, m.p. $63{ }^{\circ} \mathrm{C}$. IR ( $\left.v, \mathrm{~cm}^{-1}\right)(\mathrm{KBr}): 1625,1580,1495,1450,1390,1300,1191,820$;

${ }^{1} \mathrm{H}$ NMR (DMSO): $\delta 1.0\left(\mathrm{t}, 3 \mathrm{H},-\mathrm{CH}_{3}\right), 2.4\left(\mathrm{q}, 2 \mathrm{H},-\mathrm{CH}_{2}\right), 2.59-3.45$ (t, 8H, piperazine), 6.58.1 (m, 13H, Ar-H); Anal. Calcd for $\mathrm{C}_{25} \mathrm{H}_{26} \mathrm{~N}_{6} \mathrm{O}_{2}: \mathrm{C}, 67.89, \mathrm{H}, 5.88, \mathrm{~N}, 19.0 \%$. Found $\mathrm{C}$, $67.94, \mathrm{H}, 5.87, \mathrm{~N}, 19.01 \%$

$N$-(4-Nitrophenylimino)- $N$-(4-(4-ethylpiperazin-1-yl)phenyl)benzamidine (3d)

Yield 70\%, m.p. $127{ }^{\circ} \mathrm{C}$. IR ( $\left.v, \mathrm{~cm}^{-1}\right)(\mathrm{KBr}): 1620,1540,1500,1460,1350,1300,1250$, 1191, 850; ${ }^{1} \mathrm{H}$ NMR (DMSO): $\delta 1.0\left(\mathrm{t}, 3 \mathrm{H},-\mathrm{CH}_{3}\right), 2.4\left(\mathrm{q}, 2 \mathrm{H},-\mathrm{CH}_{2}\right), 2.59-3.45(\mathrm{t}, 8 \mathrm{H}$, piperazine), 6.6-8.2 (m, $13 \mathrm{H}, \mathrm{Ar}-\mathrm{H})$; Anal. Calcd for $\mathrm{C}_{25} \mathrm{H}_{26} \mathrm{~N}_{6} \mathrm{O}_{2}: \mathrm{C}, 67.89, \mathrm{H}, 5.88, \mathrm{~N}$, $19.0 \%$. Found C, 67.95, H, 5.89, N, $18.99 \%$

$N$-(4-Chlorophenylimino)- $N^{\prime}$-(4-(4-ethylpiperazin-1-yl)phenyl)benzamidine (3e)

Yield 75\%, m.p. $76^{\circ} \mathrm{C}$. IR (v, $\left.\mathrm{cm}^{-1}\right)(\mathrm{KBr}): 1625,1600,1484,1380,1300,1243,1089,852$;

${ }^{1} \mathrm{H}$ NMR (DMSO): $\delta 1.1\left(\mathrm{t}, 3 \mathrm{H},-\mathrm{CH}_{3}\right), 2.4\left(\mathrm{q}, 2 \mathrm{H},-\mathrm{CH}_{2}\right), 2.56-3.42(\mathrm{t}, 8 \mathrm{H}$, piperazine), 6.5$7.4(\mathrm{~m}, 13 \mathrm{H}, \mathrm{Ar}-\mathrm{H})$; Anal. Calcd for $\mathrm{C}_{25} \mathrm{H}_{26} \mathrm{~N}_{5} \mathrm{Cl}$ : C, 69.54, H, 6.02, N, 16.21\%. Found $\mathrm{C}$, $69.60, \mathrm{H}, 6.01, \mathrm{~N}, 16.22 \%$

$N$-(2-Chlorophenylimino)-N'-(4-(4-ethylpiperazin-1-yl)phenyl)benzamidine (3f)

Yield 75\%, m.p. $99{ }^{\circ} \mathrm{C}$. IR ( $\left.v, \mathrm{~cm}^{-1}\right)(\mathrm{KBr}): 1620,1590,1490,1400,1230,1090,851 ;{ }^{1} \mathrm{H}$ NMR (DMSO): $\delta 1.1\left(\mathrm{t}, 3 \mathrm{H},-\mathrm{CH}_{3}\right), 2.4\left(\mathrm{q}, 2 \mathrm{H},-\mathrm{CH}_{2}\right), 2.54-3.45(\mathrm{t}, 8 \mathrm{H}$, piperazine), 6.5-7.3 (m, $13 \mathrm{H}, \mathrm{Ar}-\mathrm{H}$ ); Anal. Calcd for $\mathrm{C}_{25} \mathrm{H}_{26} \mathrm{~N}_{5} \mathrm{Cl}: \mathrm{C}, 69.54, \mathrm{H}, 6.02, \mathrm{~N}, 16.21 \%$. Found $\mathrm{C}, 69.50$, $\mathrm{H}, 6.04, \mathrm{~N}, 16.20 \%$ 


\section{$N$-(4-Methoxyphenylimino)- $N^{\prime}-(4-(4-e t h y l p i p e r a z i n-1-y l)$ phenyl)benzamidine $(\mathbf{3 g})$}

Yield 80\%, m.p. $93{ }^{\circ} \mathrm{C}$. IR $\left(v, \mathrm{~cm}^{-1}\right)(\mathrm{KBr}): 1620,1580,1450,1400,1310,1172,1085 ;{ }^{1} \mathrm{H}$ NMR (DMSO): $\delta 1.0\left(\mathrm{t}, 3 \mathrm{H},-\mathrm{CH}_{3}\right), 2.3\left(\mathrm{q}, 2 \mathrm{H},-\mathrm{CH}_{2}\right), 2.59-3.45(\mathrm{t}, 8 \mathrm{H}$, piperazine), 3.78 (s, $\left.3 \mathrm{H},-\mathrm{OCH}_{3}\right), 6.5-7.4(\mathrm{~m}, 13 \mathrm{H}, \mathrm{Ar}-\mathrm{H})$; Anal. Calcd for $\mathrm{C}_{26} \mathrm{H}_{29} \mathrm{~N}_{5} \mathrm{O}: \mathrm{C}, 73.07, \mathrm{H}, 6.79, \mathrm{~N}$, $16.38 \%$. Found C, 73.01, H, 6.78, N, 16.35\%

\section{$N$-(2-Methoxyphenylimino)- $N^{\prime}-(4-(4-e t h y l p i p e r a z i n-1-y l)$ phenyl)benzamidine (3h)}

Yield $75 \%$, m.p. $80{ }^{\circ} \mathrm{C}$. IR $\left(v, \mathrm{~cm}^{-1}\right)(\mathrm{KBr}): 1620,1570,1450,1370,1305,1175,1085 ;{ }^{1} \mathrm{H}$ NMR (DMSO): $\delta 1.1\left(\mathrm{t}, 3 \mathrm{H},-\mathrm{CH}_{3}\right), 2.4\left(\mathrm{q}, 2 \mathrm{H},-\mathrm{CH}_{2}\right), 2.56-3.40(\mathrm{t}, 8 \mathrm{H}$, piperazine), 3.73 (s, $\left.3 \mathrm{H},-\mathrm{OCH}_{3}\right), 6.4-7.3(\mathrm{~m}, 13 \mathrm{H}, \mathrm{Ar}-\mathrm{H})$; Anal. Calcd for $\mathrm{C}_{26} \mathrm{H}_{29} \mathrm{~N}_{5} \mathrm{O}: \mathrm{C}, 73.07, \mathrm{H}, 6.79, \mathrm{~N}$, $16.38 \%$. Found C, 73.02, H, 6.80, N, 16. 40\%

\section{$N$-(4-Bromophenylimino)- $N^{\prime}$-(4-(4-ethylpiperazin-1-yl)phenyl)benzamidine (3i)}

Yield 65\%, m.p. $72{ }^{\circ} \mathrm{C}$. IR $\left(v, \mathrm{~cm}^{-1}\right)(\mathrm{KBr}): 1630,1595,1490,1380,1240,{ }^{1} \mathrm{H}$ NMR (DMSO): $\delta 1.0\left(\mathrm{t}, 3 \mathrm{H},-\mathrm{CH}_{3}\right), 2.4\left(\mathrm{q}, 2 \mathrm{H},-\mathrm{CH}_{2}\right), 2.59-3.46(\mathrm{t}, 8 \mathrm{H}$, piperazine), 6.3-7.4 (m, $13 \mathrm{H}, \mathrm{Ar}-\mathrm{H}$ ); Anal. Calcd for $\mathrm{C}_{25} \mathrm{H}_{26} \mathrm{~N}_{5} \mathrm{Br}$ : C, 63.05, H, 5.46, N, 14.70\%. Found C, 63.0, H, $5.43, \mathrm{~N}, 14.67 \%$

\section{$N$-(4-Methylphenylimino)-N'-(4-(4-ethylpiperazin-1-yl)phenyl)benzamidine (3j)}

Yield $70 \%$, m.p.82 ${ }^{\circ} \mathrm{C}$. IR $\left(v, \mathrm{~cm}^{-1}\right)(\mathrm{KBr}): 1620,1580,1450,1395,1250,{ }^{1} \mathrm{H}$ NMR (DMSO): $\delta 1.0\left(\mathrm{t}, 3 \mathrm{H},-\mathrm{CH}_{3}\right), 2.3\left(\mathrm{~s}, 3 \mathrm{H},-\mathrm{CH}_{3}\right), 2.43\left(\mathrm{q}, 2 \mathrm{H},-\mathrm{CH}_{2}\right), 2.56-3.44(\mathrm{t}, 8 \mathrm{H}$, piperazine), 6.6-7.6 (m, 13H, Ar-H); Anal. Calcd for $\mathrm{C}_{26} \mathrm{H}_{29} \mathrm{~N}_{5}: \mathrm{C}, 75.92, \mathrm{H}, 7.05, \mathrm{~N}$, $17.02 \%$. Found C, 75.87, H, 7.08, N, 16.99\%.

\section{Antimicrobial activity}

The synthesized compounds $\mathbf{3 a - 3 j}$ were screened for their antibacterial activity against $B$. Subtilis, S. Typhi and E. Coli and antifungal activity against C.albicans at a concentration of $40 \mu \mathrm{g} / \mathrm{mL}$ in DMF by cup-plate method ${ }^{18,19}$. Standard anti-bacterial and antifungal drug, gentamycin and miconazole respectively were also tested under similar conditions for comparison. Zone of inhibition in $\mathrm{mm}$ of synthesized compounds and standard drugs are shown in Table 1.

Table 1. Antimicrobial activity of compounds (3a-j).

\begin{tabular}{ccccc}
\hline Compounds & E.Coli & B.Subtilis & S.Typhi & C.Albicans \\
\hline $\mathbf{3 a}$ & 9.8 & 8.2 & 8.2 & 11 \\
3b & 7.8 & - & 7.8 & 13 \\
$\mathbf{3 c}$ & 9.2 & 10 & 7.2 & 14 \\
$\mathbf{3 d}$ & 10 & 7.4 & - & 13 \\
$\mathbf{3 e}$ & 9.4 & 7.6 & - & 12 \\
$\mathbf{3 f}$ & 12.8 & 8.6 & 9.8 & 13 \\
$\mathbf{3 g}$ & 9.6 & - & 8.4 & 14 \\
$\mathbf{3 h}$ & 9.2 & - & 8.0 & 11 \\
$\mathbf{3 i}$ & 7.8 & 8.0 & 9.8 & 11 \\
$\mathbf{3 j}$ & 9.4 & - & 10 & 12 \\
Gentamycin & 16.2 & 21.4 & 17.4 & - \\
Miconazole & - & - & - & 20 \\
\hline
\end{tabular}




\section{Conclusion}

Most of the synthesized compounds have shown antibacterial activity to some extent. Among the synthesized compounds, compound $3 \mathbf{f}$ shows good activity, while $\mathbf{3 d}, \mathbf{3 a}, \mathbf{3 g}, \mathbf{3 e}$, $\mathbf{3 j}, \mathbf{3 c}$ and $\mathbf{3 h}$ shows moderate activity. The other two compounds $\mathbf{3 b}$ and $\mathbf{3 i}$ show feeble activity against $E$. coli. Against $B$. subtilis the compound 3c shows good activity, while compounds $\mathbf{3 f}, \mathbf{3 a}, \mathbf{3 j}, \mathbf{3 e}$ and $\mathbf{3 d}$ show moderate to feeble activity. The remaining compounds $\mathbf{3 b}, \mathbf{3 i}, \mathbf{3 g}$ and $\mathbf{3 h}$ have been found to be inactive against $B$. subtilis. The compound 3j shows good activity, while compounds 3i, 3f, 3g, 3a, and $\mathbf{3 h}$ show moderate activity against $S$. typhi. Compounds $\mathbf{3 d}$ and $\mathbf{3 e}$ have been found to be inactive against $S$. typhi. The compounds $\mathbf{3 c}$ and $\mathbf{3 g}$ show good activity while the remaining compounds $\mathbf{3 b}, \mathbf{3 d}, \mathbf{3 f}, \mathbf{3 e}, \mathbf{3 j}$, 3a, $\mathbf{3 i}$ and $\mathbf{3 h}$ show moderate to good activity against $C$. albicans

\section{Acknowledgements}

The authors are thankful to the Principal, Government College, Daman for providing necessary research facilities and Konark Research Foundation, Daman for spectral analysis and biological screening.

\section{References}

1. Pandey V K and Negi H S, Indian Drugs (Indian Drug Manufacturers Association), 1999, 36(1), 37.

2. Mishra V S, Dhar S and Chowdhary B L, Pharmazie, 1978, 33, 790.

3. Desai R M and Desai J M, Indian J Heterocycl Chem., 1999, 8(4), 329.

4. Garg H G and Kaur M, J Med Chem., 1992, 15, 554.

5. Desai K G and Desai K R, Indian J Chem., 2005, 44(B), 2097.

6. Bhardwaj S D, Phatak P and Jolly V S, Orient J Chem., 1995, 2, 181.

7. Venkal N K, J Med Chem., 1998, 8, 11.

8. Bhardwaj S D and Jolly V S, Asian J Chem., 1997, 9, 48.

9. Desai J M and Shah V H, Indian J Chem., 2003, 42(B), 631.

10. Khanna R, Saxena A K, Srivastava V K and Shanker K, Indian J Chem., 1990, 29(B), 91.

11. Kumar P, Nath C, Agarwal J C, Bhargava K P and Shanker K, Indian J Chem., 1983, 22(B), 955.

12. Naithani P K, Srivastava V K, Barthwal J P, Saxena A K, Gupta T K and Shanker K, Indian J Chem., 1989, 28(B), 990.

13. Kumar P, Nath C and Shanker K, Pharmazie, 1985, 40, 267.

14. Patel N B and Bhagat P R, Asian J Chem., 2003, 12, 83.

15. Kulkarni S S and Jianjing C, J Med Chem., 2003, 46, 2589.

16. Corrode M, Tuxi D G and Ungersteadt, J Pharmacol., 1971 23, 983.

17. Vogel A I, A Textbook of Practical Organic Chemistry, $5^{\text {th }}$ Ed., Longmann Group Ltd., London, 921, 1989.

18. Banty A L, The Antimicrobial Susceptibility test; Principle and practice, Edited by Illus lea and Febiger, (Philadelphia, Pa USA), 1976, 180.

19. Seely H W and Van Demark P J, Microbes in action: A laboratory manual of Microbiology, D B Taraporewala Sons and Co, Bombay, 1975, 55-80. 


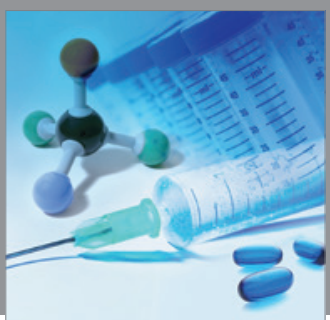

International Journal of

Medicinal Chemistry

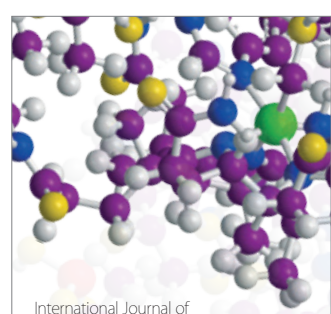

Carbohydrate Chemistry

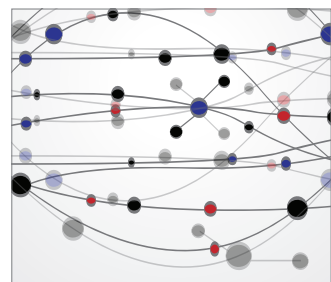

The Scientific World Journal
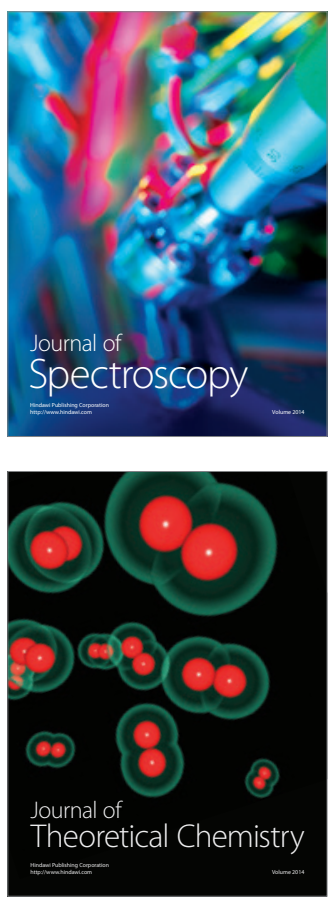
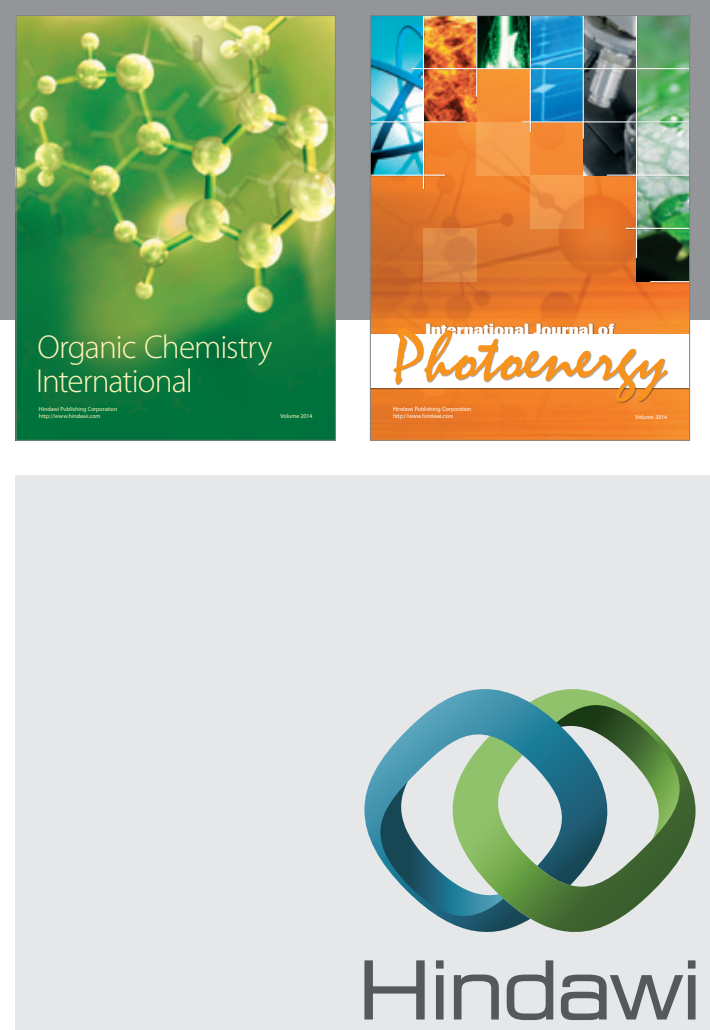

Submit your manuscripts at

http://www.hindawi.com
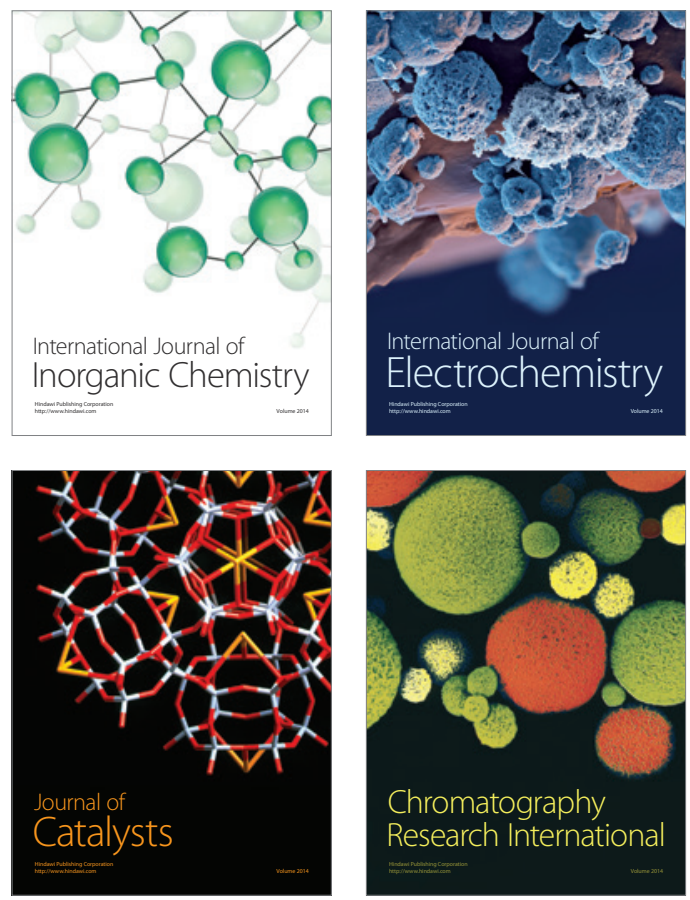
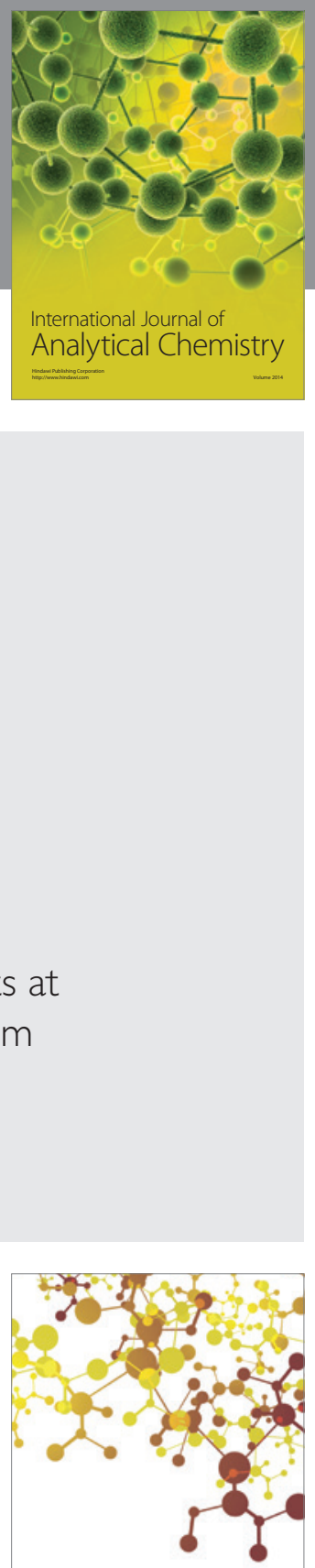

Journal of

Applied Chemistry
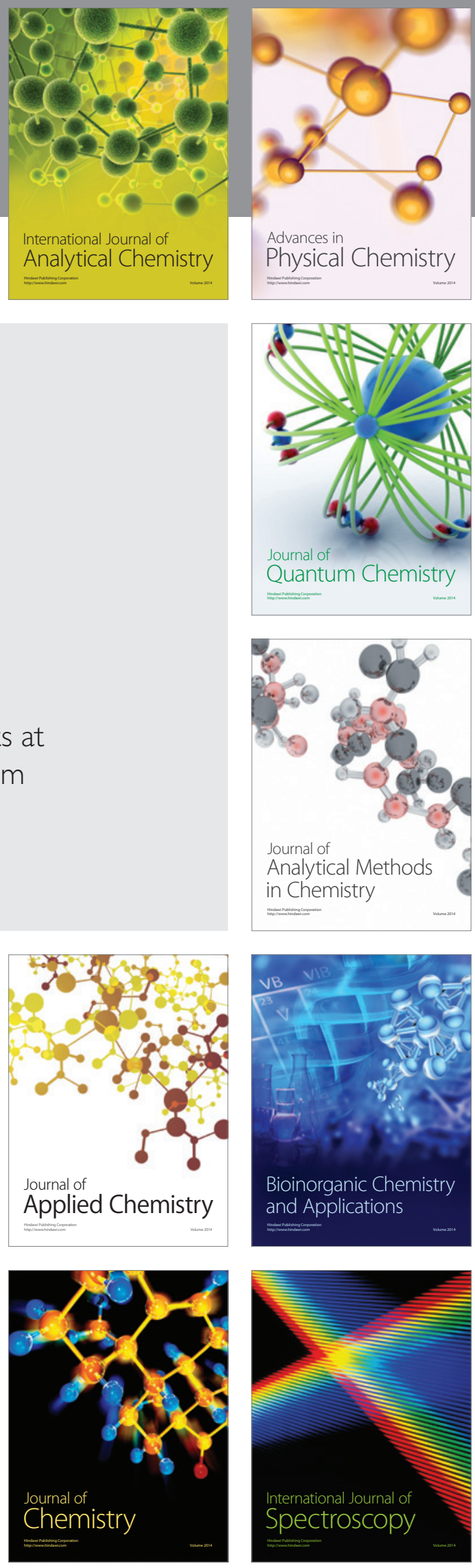